\title{
Hiperidrose - Conceitos e Atualizações Cirúrgicas
}

\author{
Professor Mestre Mauro Tavares (Ciências da Saúde - UniFOA) - \\ mauro.tavares@snt.com.br \\ Aluna Juliana Sobreira Furtado Silva(Ciências da Saúde - UniFOA) - \\ juju.furtado@gmail.com \\ Aluna Polyanna Pereira de Azevedo(Ciências da Saúde - UniFOA) - \\ poly.azevedo1@gmail.com
}

\section{Resumo}

A hiperatividade do sistema nervoso autônomo simpático leva a um aumento da atividade das glândulas sudoríparas que em resposta a este estímulo, promove uma transpiração excessiva, através de uma sudorese constante, sendo esta condição conhecida como hiperidrose. Com incidência de 0,5 a 1\% da população, a hiperidrose pode ser primária ou secundária e pode atingir mãos, pés, face e couro cabeludo. Pode ser submetida a tratamento clínico ou cirúrgico, sendo esta última modalidade de tratamento, a única forma definitiva no controle da hiperidrose. Contudo, um dos efeitos mais indesejáveis no pós-operatório, é a sudorese reflexa, que segundo alguns autores pode acometer $85 \%$ dos pacientes submetidos à ressecção da cadeia simpática. A incidência e a intensidade da sudorese reflexa é de causa multifatorial, podendo depender: do peso, sexo, idade e do(s) nível(eis) de secção(ões) em que os pacientes serão submetidos, de acordo com o tipo de hiperidrose que os mesmos apresentarem. Novas abordagens cirúrgicas da cadeia simpática estão sendo propostas como forma á minimizar a sudorese reflexa no pós-operatório, levando-se em consideração todos os fatores preditivos comentados na bibliografia científica.

\section{Introdução}

O controle da temperatura corpórea é feito através do suor, com destaque para situações de atividade física, quando, por exemplo, o ambiente se encontra em temperaturas mais elevadas ou o individuo se encontra submetido a alterações emocionais. A intensidade da sudorese vai variar dependendo de fatores como sexo, idade, raça, além de fatores endógenos e exógenos. O sistema nervoso autônomo simpático é responsável pela regulação da sudorese corpórea. A hiperatividade desse sistema leva a um aumento da atividade das glândulas sudoríparas que em resposta a este estímulo, promove uma transpiração excessiva, através de uma sudorese constante, sendo esta condição conhecida como hiperidrose. Sua incidência acomete 0,5 a $1 \%$ da população, sendo considerada uma condição clínica freqüente (COELHO, 2006). A hiperidrose pode ser primária ou secundária. A hiperidrose primária não tem causas conhecidas, se devendo mais a fatores genéticos. As pessoas simplesmente nascem com uma tendência maior a desenvolver a hiperidrose em determinadas áreas do corpo, que pode se manifestar logo nos primeiros anos de vida, ou em qualquer fase posterior. A hiperidrose secundária está relacionada a uma doença de base como, por exemplo, o hipertireoidismo, distúrbios psiquiátricos, menopausa ou a obesidade. A hiperidrose pode atingir axilas, as mãos(Fig. 1) e os pés, mas pode atingir também a face, principalmente a região frontal(testa) e o couro cabeludo, assim como o tórax, a nuca, a região sob a mama e a região inguinal. 


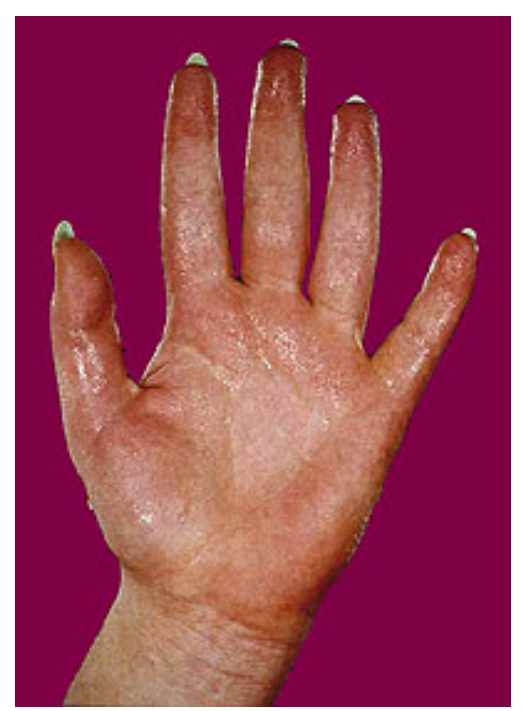

Fig. 1 - Hiperidrose palmar

Esta é uma condição clínica extremamente desagradável levando a limitações na atividade profissional dos indivíduos, além de interferir no desenvolvimento de suas atividades sociais, e até mesmo em seus relacionamentos. Em quadros mais severos de hiperidrose, o indivíduo pode apresentar gotejamento espontâneo na região afetada, que pode ser acompanhada por maceração e fissuração da pele. E se esta sudorese se torna mais intensa, como por exemplo, na região axilar, outros sintomas desagradáveis podem ocorrer, destacando-se o odor fétido no local afetado. Isso é chamado de bromidrose, e está relacionado com a presença de fungos e bactéria na pele, que ao contato com o suor, promovem o odor (LEÃO, 1999). O tratamento pode ser clínico ou cirúrgico. O primeiro tem como destaque o uso da toxina botulínica (Botox). Esta pode ser aplicada nas mãos, nas axilas e em outros locais, eliminando temporariamente o suor. Em função desta característica, o tratamento clínico deve ser por tempo indeterminado, o que acaba acarretando um custo elevado ao mesmo (COELHO, 2006). O tratamento cirúrgico é um tratamento efetivo para hiperidrose, e tem como principal conduta a simpaticotomia. Conceitualmente, a simpatectomia difere da simpaticotomia, pelo fato de que na primeira retira-se parte da cadeia simpática, ao passo que na simpaticotomia procede-se apenas à secção da cadeia. A simpaticotomia, que hoje é amplamente realizada por videotoracoscopia, ganhou destaque nos últimos anos, sendo considerada o tratamento de escolha para casos de hiperidrose palmar, axilar, facial e em casos associados, incluindo a hiperidrose plantar. Outras indicações para a ablação cirúrgica da cadeia simpática torácica são no auxílio ao tratamento da: distrofia simpática reflexa, em casos selecionados de isquemia grave de membro (doença vascular periférica embólica ou aterosclerótica), doença de Raynaud, síndrome do QT longo e causalgia. Porém os melhores resultados para esta técnica envolvem o tratamento da hiperidrose primária (COELHO, 2006). A simpaticotomia está contra-indicada em pacientes que: possuem hiperidrose secundária, em indivíduos portadores de insuficiência respiratória ou cardiovascular devido à impossibilidade de ventilação adequada durante o ato cirúrgico, em pacientes com seqüela de doença pleural, como tuberculose ou empiema pleural, devido a maiores chances de complicações na operação, como o pneumotórax. O índice de massa corpórea é um dado importante que, atualmente, tem levado os cirurgiões de tórax a repensarem as indicações cirúrgicas quando o mesmo é maior que 25, pois os resultados se tornam ruins. Isso ocorre porque a hiperidrose de um paciente com sobrepeso, ou obeso, que, á princípio, se acredita que a mesma seja primária, na realidade é do tipo secundária, devendo ser contra-indicada a operação, nestes casos. O efeito colateral mais comum dessa cirurgia é a 
sudorese compensatória, ou sudorese reflexa, que pode ocorrer no tronco, e em membros inferiores, principalmente quando o individuo que passou pelo procedimento cirúrgico se encontra em ambientes quentes e após a realização de exercícios físicos, e consiste de um suor exagerado em locais do corpo que não foram denervados pela simpatectomia (LEÃO,1999). Com isso, a maioria dos cirurgiões passou a se preocupar com uma cirurgia menos extensa e mais seletiva. Ao invés de proceder a uma simpatectomia, passaram a fazer somente a simpaticotomia (Fig. 3). Com secção da cadeia simpática torácica, somente no(s) nível(eis) relacionados aos casos da hiperidrose primária. Quanto menos extensas são as secções da cadeia simpática, menor será a intensidade da hiperidrose reflexa. Outra modalidade cirúrgica para tratar a hiperidrose primária, é a utilização somente de clipes metálicos; justamente por permitir a remoção dos mesmos, caso a insatisfação com os resultados da operação, sejam elevadas (FURIAN, 2006).

\section{Fundamentação Teórica}

A anatomia do sistema nervoso (SN) humano pode ser dividida em sistema nervoso somático e sistema nervoso visceral. O primeiro é também denominado sistema nervoso de vida de relação, ou seja, é aquele que relaciona o indivíduo com o meio. Para isto, a parte aferente do sistema nervoso somático conduz aos centros nervosos impulsos originados em receptores periféricos, informando estes centros sobre o que se passa no meio ambiente. $\mathrm{O}$ sistema nervoso visceral, ou de vida vegetativa, relaciona-se com as estruturas viscerais e é muito importante para a integração das atividades das vísceras no sentido da manutenção da constância do meio interno (homeostase). Ambos os sistemas são constituídos de fibras aferentes (sensitivas) e fibras eferentes, ou motoras. Por definição, denomina-se sistema nervoso autônomo (SNA) apenas o componente eferente do sistema nervoso visceral. O sistema nervoso autônomo pertence à porção periférica do $\mathrm{SN}$ e se divide em simpático e parassimpático. Parte do SN somático se origina, em parte, no tronco cerebral - são os chamados nervos cranianos - que vão fazer a inervação da cabeça, face e pescoço. Ele também pode ser originado na medula espinhal, de onde emergem fibras somáticas que vão se dirigir ao tronco e membros. Essas fibras, que saem das fibras ventrais da medula espinhal, são chamadas de nervos espinhais, ou raquidianos. Elas inervam os músculos esqueléticos, onde estão envolvidas com a função muscular esquelética. As fibras autônomas, no conjunto que forma o SNA, vão ter como papel funcional inervar as vísceras (coração, glândulas e estruturas que contém músculo liso). Normalmente as divisões somática e visceral funcionam em conjunto (associação) com o objetivo de ajustar o nosso organismo às alterações do meio ambiente. Com isso, faz-se necessário á ativação do sistema parassimpático ou do sistema simpático. O SN parassimpático tem origem na divisão crânio-sacral, pois os corpos celulares das fibras pré-ganglionares parassimpáticas se originam em áreas e núcleos motores de certos nervos cranianos presentes no tronco cerebral ou segmento sacromedular, S2 à S4. O SN simpático é também chamado de divisão tóraco-lombar (Fig. 2), pois suas fibras préganglionares se originam no segmento tóraco-lombar, que se estende de T1 até L2 ou L3. A porção periférica desse sistema é formada por duas cadeias de gânglios paravertebrais nos dois lados da coluna (CARPENTER, 1991). 


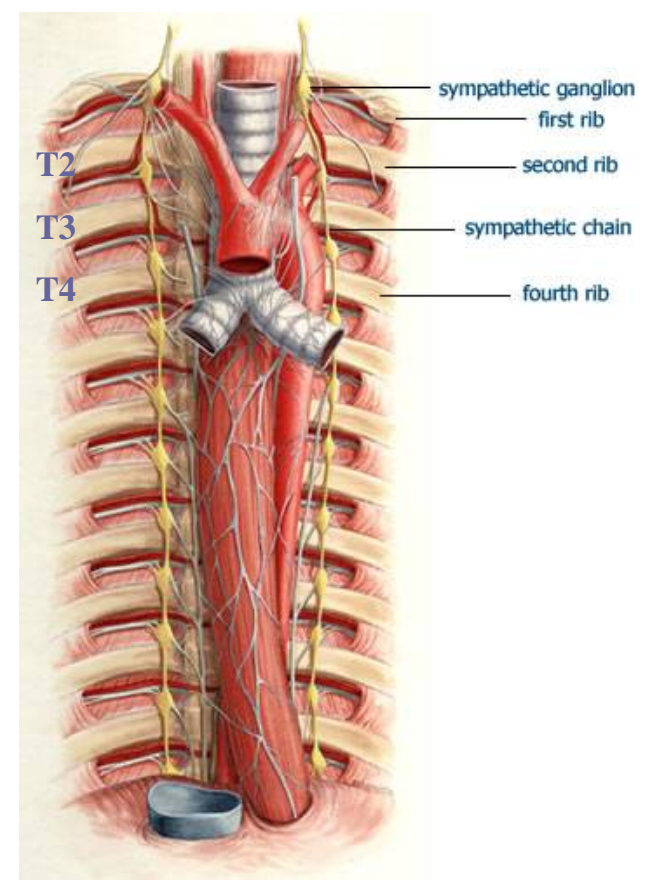

Fig. 2 - Segmento torácico da cadeia simpática

Na hiperidrose ocorre à exacerbação do reflexo simpático colinérgico (fibra préganglionar), responsável pela excitação das glândulas sudoríparas. Existem dois tipos de glândulas na pele, as apócrinas e as écrinas. As glândulas sudoríparas apócrinas têm um papel menor na térmo-regulação (regulação da temperatura corporal), estão associadas ao folículo piloso e não estão envolvidas no processo de hiperidrose. A hipersecreção das glândulas écrinas é que causam as alterações observadas. As glândulas écrinas estão mais concentradas nas axilas, palmas e plantas e tem importante papel na térmo-regulação. O excesso de secreção desta glândula é que causa a hiperidrose. Existem de 2 a 5 milhões de glândulas écrinas distribuídas por todo o corpo. O sistema autônomo, em situações de calor intenso, ansiedade, fortes emoções, assume um comportamento parecido com o do sistema nervoso endócrino, no qual ocorre a regulação, via feedback, dos centros térmo-reguladores do hipotálamo, que seguem por via simpática colinérgica a direção eferente do reflexo até a periferia corporal, resultando na perda de calor através do suor (GUYTON, 1981). As simpatectomias provocam uma ablação no gânglio nervoso na coluna paravertebral ganglionar, e quanto mais alto for o nível da ablação, maior é o efeito bloqueador de estímulos aferentes ao hipotálamo. Sendo assim, uma secção em níveis mais baixos da coluna paravertebral, permite que continuem chegando estímulos periféricos suficientes ao hipotálamo, evitando o principal efeito colateral das simpaticotomias, que é a hiperidrose reflexa, também chamada sudorese compensatória. Em níveis altos de ablação, ocorre um intenso reflexo de hiperidrose devido ao bloqueio severo das vias aferentes para o hipotálamo. O primeiro gânglio simpático torácico (T1) é responsável pela maioria do suor e perda do calor da face, das mãos e em menor extensão das axilas. O T1, juntamente com o oitavo gânglio cervical (C8) constituem o Gânglio Estrelado, que é responsável pela inervação das pálpebras e resposta pupilar (área orbital), devendo ser preservados na simpatectomia, pois podem causar, quando lesados, a Síndrome de Claude Bernard Horner. O segundo gânglio torácico (T2) controla a resposta sudorífera das mãos e da face (com exceção da porção intraorbital), couro cabeludo, ombros e parte anterior e posterior do tórax acima dos mamilos e o rubor facial (blushing) . O terceiro gânglio torácico (T3) afeta a sudorese das mãos, das axilas, 
ombros e parte anterior e posterior do tórax acima dos mamilos e, em menor grau, da face. O quarto gânglio torácico (T4) inerva as mãos e as axilas. Como se percebe, pelo escrito acima, há uma superposição de inervação simpática para a face, mãos e axilas (COELHO, 2006). De acordo com este autor, tradicionalmente, a simpatectomia é realizada ao nível de T2 para hiperidrose crânio-facial e rubor facial, T3 para hiperidrose palmar, T3 e T4 para hiperidrose axilar associada ou não a hiperidrose palmar. Porém os efeitos relacionados a hiperidrose compensatória se sobressaíram em grande escala, levando pacientes a se arrependerem da realização da cirurgia, queixando-se que a sudorese compensatória incomodava mais do que a hiperidrose inicial, anterior à cirurgia.

Os resultados da simpatectomia foram divididos em:

EXCELENTE: onde a região operada esta completamente seca.

BOM: a região ainda apresenta sudorese discreta.

REGULAR: melhora parcial da sudorese.

RUIM: que não houve melhora alguma.

Com isso, pode-se verificar que em relação a hiperidrose palmar e a crânio-facial, tivemos 99,5\% e 90,9\%, respectivamente, de resultados excelentes e bons. Na hiperidrose axilar $90,7 \%$ tiveram resultados bons e excelentes. A taxa de remissão da hiperidrose plantar associada foi de 32\%, sendo que 29,7\% tiveram melhora parcial e 38,3\% não tiveram nenhuma melhora após dois anos de observação clínica. A literatura médica mundial mostra 94-100\% de cura da hiperidrose palmar e/ou axilar, sendo permanente. A explicação de alguns autores para o fato da hiperidrose axilar apresentar índices menores de sucesso terapêutico $(90,7 \%)$ é o fato de que na região axilar existirem $90 \%$ de glândulas sudoríparas écrinas e $10 \%$ de glândulas sudoríparas apócrinas. As glândulas écrinas são dependentes do simpático, e quando são feitas as simpatectomias elas param de secretar suor. Já as glândulas apócrinas não são estimuladas pelo simpático, portanto não são influenciadas pela simpatectomia (COELHO, 2006). Segundo trabalho de LYRA (2006), as cirurgias são na maioria das vezes curativa: $90 \%$ apresentam melhora na hiperidrose palmar, axilar, facial, couro cabeludo e blushing, e em 85\% desses pacientes obtém alguma melhora na hiperidrose plantar. Os membros superiores e axilas encontram-se secos e quentes em 95\% dos casos. Em $70 \%$ das vezes, o mesmo ocorre em relação a hiperidrose plantar e craniofacial. Foi relatado que em relação às taquicardias e palpitações, houve resultados permanentes positivos. Entretanto, em 20 a 50\% dos pacientes, ocorreu hiperidrose compensatória, provavelmente por uma resposta térmo-reguladora (GOSSOT, 2003), sendo esta condição tolerável para a maioria dos pacientes; e somente $10 \%$ queixaram-se desta perspiração excessiva, mas a toleram melhor que a sudorese palmar. O acompanhamento prolongado dos pacientes indica que $95 \%$ a $99 \%$ deles experimentaram alívio do suor nas mãos. Noventa a 95\% apresentaram alivio do suor axilar e do suor e rubor crâniofacial. Por fim, cerca de $8 \%$ ficaram insatisfeitos com o resultado final, mas apenas $4 \%$ deles se arrependeram de terem sido submetidos à operação. No Medical City Dallas Hospital, Texas, DEWEY e cols.(2006), realizaram a simpatectomia por toracoscopia em 222 pacientes entre janeiro de 2002 e novembro de 2003. Desses, 170 pacientes foram acompanhados uma vez por ano. Nesse tempo, foram analisados os resultados obtidos pós-simpatectomia, sendo relatado que as áreas que apresentavam hiperidrose, todas tiveram melhoria significativa. Entretanto, a hiperidrose compensatória foi relatada em $85 \%$ dos pacientes acompanhados a mais de um ano. Além disso, nos pacientes em que a simpatectomia foi ao nível de T2 a hiperidrose compensatória foi mais severa que naqueles em que a simpatectomia foi realizada em outros níveis, em que não houve relato de hiperidrose compensatória, e com alto grau de satisfação por parte dos pacientes. Com os resultados obtidos através da realização da simpatectomia ao nível de T2, observou-se que os efeitos da sudorese compensatória poderiam estar associados com a extensão da denervação simpática. Nos trabalhos de RIET (2001) e LIN (2001), há um conceito atual, porém não 
unânime, de que T2 manteria a maioria dos estímulos aferentes ao centro sudomotor situado no hipotálamo. Portanto, a sudorese seria reflexa, e não compensatória como afirmam alguns autores (COELHO, 2006). De acordo com os resultados da experiência clínica adquirida, LIN e col.(2001) propuseram tratar as diferentes órdens do simpático por diferentes cirurgias, com o objetivo de selecionar melhor a cirurgia e minimizar os efeitos colaterais. Com isso, foi criada a classificação Lin-Telaranta para as desordens do simpático e suas respectivas propostas cirúrgicas:

Grupo 1: cirurgia ao nível de T2. Indicada para problemas cerebrais como: vermelhidão facial, rinite, desordens psíquicas, angina pectoris, hipertensão, tremores, parkinson, enxaqueca, dependência a drogas e doenças do sono.

Grupo 2: cirurgia ao nível de T3. Indicada para problemas da cabeça à clavícula como: hiperhidrose crânio-facial, com ou sem vermelhidão facial, hiperhidrose crânio-facial associada a hiperhidrose palmar, rosácea, angina pectoris, tremores, Parkinson e enxaqueca.

Grupo 3: cirurgia ao nível de T4. Indicada para problemas da clavícula ao mamilo: hiperhidorse palmar e/ou axilar (FURIAN, 2006).

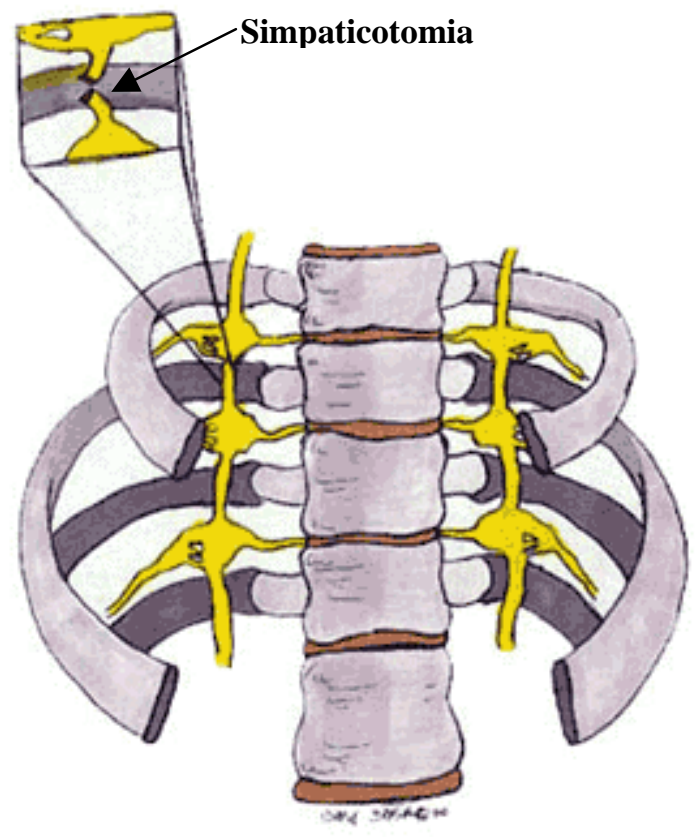

Fig. 3 - Detalhe da simpaticotomia

Em agosto de 2001, RIET e cols. realizaram uma série de cirurgias envolvendo 14 pacientes com hiperidrose das mãos e/ou axilas. Ao invés de realizarem a tradicional simpatectomia, com a ressecção ou cauterização do gânglio, foi realizado a simpaticotomia (somente secção da cadeia simpática) cranial e caudal ao gânglio T3. A cirurgia foi eficaz em todos os pacientes. Em relação à incidência de hiperidrose compensatória resultante, nenhum 
paciente deste estudo apresentou hiperidrose compensatória pós-operatória. Diferentemente da maioria das técnicas preconizadas até então, o nível da cirurgia foi seletivado caudalmente (T3 no lugar de T2) e o gânglio alvo foi preservado (FURIAN, 2006).

Em 2001, LIN e cols. propuseram o uso de clipes metálicos para o tratamento da hiperidrose, promovendo assim um bloqueio da cadeia simpática. Com isso, a cirurgia passou a ser vista como um processo reversível, no caso de insucesso ou arrependimento do paciente. O uso dos clipes metálicos também proporciona uma confirmação radiológica do bloqueio pós-operatório. Isso é valorizado pelo fato de constantes erros em relação ao nível cirúrgico pretendido, cometido até mesmo por cirurgiões experientes. E foi exatamente devido a um erro anatômico que LIN realizou a clipagem cranial e caudal ao nível de T4 em um paciente, e verificou que não houve nenhum grau de sudorese reflexa. Assim, ele mudou o alvo da cirurgia (anteriormente ao nível de T2), passou a realizar o isolamento do gânglio de T4 em 165 pacientes com hiperidrose palmar e/ou axilar, tratados com clipagem da cadeia simpática. Ele teve como resultado que nenhum paciente apresentou hiperidrose reflexa pós-operatória, sendo a cirurgia eficaz em todos estes pacientes, com exceção de um (FURIAN, 2006). No Brasil, FURIAN, M.B, após o conhecimento dessa nova abordagem para o tratamento da hiperidrose, passou a realizar a cirurgia tendo como alvo o gânglio T4, classificando o resultado da cirurgia em T4 como algo mais "fisiológico". Porém, o uso de clipes metálicos ficou limitado devido à inexperiência do próprio colega com a clipagem e a dificuldade em obter os clipes específicos em formato de "U”, além do custo adicional em se obter clipes e grampos, optando, então, pela simpaticotomia. Em maio de 2002, FURIAN realizou a ressecção simpática ao nível de T2 e T3 em 64 pacientes que apresentavam hiperidrose axilar e/ou palmar. Destes, 56 apresentaram hiperidrose compensatória (87,4\%). Dos pacientes que apresentaram hiperidrose compensatória, cinco a consideraram insuportável, classificando-a como pior do que o problema original, alegando ainda que o problema piorava com o calor. $\mathrm{O}$ restante dos pacientes revelou que a intensidade do suor reflexo havia diminuído nas primeiras semanas, atingindo um nível tolerável. Porém, havia momentos de exacerbação nos períodos de calor intenso ou na realização de exercícios físicos. No período de maio de 2002 a março de 2004, FURIAN passou a realizar a simpaticotomia ao gânglio T4, e após operar 244 pacientes apresentando, obteve o seguinte resultado: a cirurgia foi eficaz em 240 pacientes, sendo que apenas 93 pacientes (38,1\%) apresentaram algúm grau de hiperidrose reflexa; porém, nenhum deles a classificou como intolerável, referindo que o suor reflexo aparecia quando estava muito quente, durante exercícios físicos e em situações de estresse, não sabendo afirmar se aquela situação deveria ser considerada anormal (FURIAN, 2006). 


\section{Conclusão}

Embasado nos artigos pesquisados, é factível a simpaticotomia como forma definitiva no tratamento da hiperidrose. Contudo, não podemos deixar de registrar que a hiperidrose reflexa é um fato real e que deva ser encarado com muita preocupação, pois a mesma poderá atingir até $87 \%$ dos pacientes. Um trabalho exposto por ALMEIDA, C. A. no Congresso Norte-Nordeste Cirurgia Torácica, de maio de 2006, o autor enfatizou vários fatores preditivos para hiperodrose reflexa, entre eles: o índice de massa corpórea, a idade e o sexo. O índice de massa corpórea (IMC) é um fator relacionado diretamente com a satisfação dos pacientes, ou seja, quanto maior o IMC maior será a incidência de hiperidrose reflexa, e maior será a insatisfação dos pacientes; talvez por se tratar de uma hiperidrose secundária e não primária. Ainda segundo este autor, a idade acima de 32 anos e o sexo masculino, seriam fatores preditivos negativos na indicação cirúrgica para o tratamento da hiperidrose.

Por fim, todos estes dados expostos acima, nos levam a repensar os critérios de indicação cirúrgica para o tratamento da hiperidrose. Acreditamos que estudos prospectivos, triplo-cego e randomizados, com sólidas fundamentações metodológicas, deverão ser excutados, no afã de esclarecer e propor técnicas para se evitar, cada vez, mais a hiperidrose reflexa, e otimizar, assim, os resultados positivos tão almejados tanto pelos cirurgiões quanto pelos pacientes.

\section{Referências Bibliográficas}

ADAR, R, KURCHIN, A, ZWEIG, A, MOZES, M - Palmar hyperhidrosis and its surgical treatment: a report of 100 cases. Ann. Surg., 1977; 186(1):34-41.

CARPENTER, M.B. Core Text of Neuroanatomy. Baltimore, Ed. Williams \& Wilkins, 1991.

COELHO,M.Hiperhidrose.Paraná,jun2006.http://www.marloscoelho.com.br/conteudo_hiper hidrose.php?acao=hiperhidrose\&area=hiperhidrose_completa\&idioma=1] Acesso em 12 de junho 2006

CHIOU, T.S., CHEN, S.C. Intermediate-term results of endoscopic transaxillary T2 sympathectomyfor primary palmaris hyperhidrosis. Br Jour Surg 86: 45-47, 1999

DEWEY,T.M, HEBERT,M.A., HILL.S.L., PRINCE S.L. e MACK, M.J One year follow-up after thoracoscopic sympathectomy for hiperhidrosis: outcomes and consequences. Ann Thorac Surg - April . Texas, EUA, 2006

FURIAN, M.B. Simpatectomia Videotoracoscópica e Hiperhidrose Reflexa Pós Operatória. Boletim Científico da Sociedade Brasileira de Cirurgia Torácica, ano II n.4,

GOSSOT, D., GALLETA, D., PASCAL, A. et al. Lond-Term results of endoscopic thoracic sympathectomy for upper limb hyperhidrosis. Ann Thorac Surg 75: 1075-9, 2003. 
GUYTON, A.C. Fisiologia Humana. 5ª ed., Rio de Janeiro, Ed. Interamericana, 1981.

KAO, M.C, LIN, J.Y, CHEN, Y.L, HSIEH, C.S, CHENG, L.C, HUANG, S.J. - Minimally invasive surgery: video endoscopic thoracic sympathectomy for palmar hyperhidrosis. Ann. Acad. Med. Singapore, 1996; 25(5):673-678.

LEÃO, L.E.V.; GIUDICI,R. Simpatectomia Torácica por Videotoracoscopia: tratamento atual da hiperhidrose palmar. Revista Jovem Médico, maio 1999.

LIN, C.C., WU, H.H. Endoscopic T4-Sympathectomy block by clamping(ESB4) in treatment of hyperhidrosis palmaris et axillaris - experiences of 165 cases. Annales Chirurgiae et Gynaecologiae 90:167-169, 2001

LIN, C.C., TELERANTA, T. Lin-Telaranta classification: The importance of different procedures for different indications in sympathetic surgery. Annales Chirurgiae et Gynaecologiae 90: 161-166, 2001

LYRA, R.B. e SALVESTRO, J.E.S. Informação aos pacientes com transpiração excessiva. São Paulo, 2006 [http://www.geocities.com/manuseio/hiperidrose.html] Acesso em 12 de junho 2006.

RIET, M., SMET, A., KUIKEN, H, KAZEMIER, G., BONJER, H.J. Prevention of compensatory hyperhidrosis after thoracoscopic sympathectomy for hyperhidrosis. Surg Endosc 15:1159-1162, 2001.

URSCHEL, H.C. Dorsal sympathectomy and manegement of thoracic outlet syndrome with VATS. Ann Thorac Surg 1993;: 56:717-20.

VALLIÈRIS, E. Endoscopic Upper Thoracic Sympathectomy. Neurosurgery Clinics of North América 12:321-327, 2001.

ZACHERL, J., HUBER, E.R, IMHOF, M. et al. Long-term results of 630 thoracoscopic sympathicotomies for primary hyperhidrosis: The Vienna experience. Eur J Surg Suppl, 1998,: 580, 43-6.

Informações bibliográficas:

Conforme a NBR 6023:2002 da Associação Brasileira de Normas Técnicas (ABNT), este texto científico publicado em periódico eletrônico deve ser citado da seguinte forma: SOUZA, M. C. T.; SILVA, J. S. F.; AZEVEDO, P. P. . Hiperidrose - Conceitos e Atualizações Cirúrgicas. Cadernos UniFOA, Volta Redonda, ano 1, nº. 1, jul. 2006. Disponível em: <http://www.unifoa.edu.br/pesquisa/caderno/edicao/01/113.pdf> 